\title{
Notice nécrologique \\ AUGUSTE PRENANT
}

Le décès du professeur Auguste Prenant, survenu en octobre 1927, est une très grande perte pour la science.

Louis-Auguste Prenant, né à Lyon en 1861, fit ses études médicales à Nancy et présenta, en 1887, sa thèse de doctorat sur la structure du tube séminifère des mammifères. Agrégé en 1892, il fut nommé, en 1894, professeur à la Faculté de médecine de Nancy et, en 1907, professeur à la Faculté de médecine de Paris. En 1911, il était élu membre de l'Académie de médecine.

Le professeur A. Prenant laisse une œuvre scientifique considérable dans les divers domaines de la Biologie. A coté de ses travaux sur le limaçon, sur les cellules trachéales, sur le muscle, qui sont du domaine de la morphologie, les recherches sur l'appareil pinéal et sur le développement des dérivés branchiaux appartiennent à l'embryologie. Mais c'est particulièrement en histologie et en cytologie que s'est manifestée l'activité inlassable et féconde du savant, sur les sujets les plus divers: les recherches sur la spermatogénèse, la découverte du corpuscule intermédiaire, l'introduction de la notion nouvelle de la préspermatogénèse, les travaux sur les appareils ciliés et leurs dérivés, la démonstration histologique de l'existence de processus glandulaires dans le corps jaune, la mise en évidence de l'origine mitochondriale fréquente des grains de pigments, enfin la notion du protoplasma supérieur et de l'ergatoplasma, notion qui fut un stimulant important pour toute une série de recherches.

$\mathrm{Au}$ point de vue de la technique, A. Prenant a laissé des méthodes de fixation et de coloration qui sont tout à fait classiques. Ses deux ouvrages, le traité d'embryologie et le traité de cytologie, publié en collaboration avec Bourn et MaILIARD, sont des cuvres didactiques considérables dont l'influence heureuse a été très grande sur le développement de l'histologie et de la cytologie.

L'œuvre du grand savant fut complétée par celle du maître. A. Prenast fut, avant tout, un chef d'école et la série de brillants élèves, éduqués dans son Iaboratoire, élèves dont plusieurs sont aujourd'hui des maîtres réputés, constitue la preuve la plus frappante de l'apport considérable que cette belle figure de chercheur et de guide a apporté aux progrès de la science.

Les conceptions de A. Prenant sur l'orientation des recherches cytologiques vers l'histophysiologie et l'histochimie sont en trop parfaite concordance avec les idées que la Revue Protoplasma essaye de répandre pour que la disparition d'un collaborateur aussi éminent ne constitue pour la Revue une perte particulièrement pénible.

Ph. Joyet-Lavergne. 